Michael Trott

\title{
The Mathematica GuideBook for Numerics
}

With 1364 Illustrations 


\section{Contents}

0. Introduction and Orientation xvii

\section{CHAPTER 1}

\section{Numerical Computations}

\subsection{Remarks 1}

1.1 Approximate Numbers 15

1.1.0 Remarks 15

1.1.1 Numbers with an Arbitrary Number of Digits 15

1.1.2 Interval Arithmetic 54

1.1.3 Converting Approximate Numbers to Exact Numbers 66

1.1.4 When N Does Not Succeed 94

1.1.5 Packed Arrays 101

1.2 Fitting and Interpolating Functions 124

1.3 Compiled Programs 159

1.4 Linear Algebra 203

1.5 Fourier Transforms 225

1.6 Numerical Functions and Their Options 264

1.7 Sums and Products 272

1.8 Integration 289

1.9 Solution of Equations 316

1.10 Minimization 347

1.11 Solution of Differential Equations 368

1.11.1 Ordinary Differential Equations 368

1.11.2 Partial Differential Equations 451 
xvi

\subsection{Two Applications 481}

1.12.0 Remarks 481

1.12.1 Visualizing Electric and Magnetic Field Lines 481

1.12.2 Riemann Surfaces of Algebraic Functions 508

Exercises 521

Solutions 703

References 915

CHAPTER 2

Computations with Exact Numbers

2.0 Remarks 969

2.1 Divisors and Multiples 974

2.2 Number Theory Functions 1009

2.3 Combinatorial Functions 1030

2.4 Euler, Bernoulli, and Fibonacci Numbers 1046

Exercises 1060

Solutions 1076

References 1173

Index 1191 